Journal of

Epilepsy and

Clinical

Neurophysiology

J Epilepsy Clin Neurophysiol 2008; 14(1):11-16

\title{
Valor Prognóstico do EEG Neonatal em Recém-Nascidos de Alto Risco
}

\author{
Richard Lester Khan*, Júlia de la Puerta Raya**, Maria Carolina Ghellar Fürst**, \\ Magda Lahorgue Nunes*** \\ Serviço de Neurologia e Laboratório de Neurofisiologia Clínica do Hospital São Lucas da PUCRS
}

\begin{abstract}
RESUMO
Introdução: A detecção precoce de disfunção ou insulto cerebral em recém-nascidos de risco internados dentro das unidades de terapia intensiva neonatal aumenta a possibilidade de melhor sobrevida e de menor risco de seqüela neurológica destas crianças. O EEG é uma ferramenta que possibilita ao neonatologista uma informação da repercussão da lesão neurológica no cérebro imaturo e, junto aos achados de neuroimagem, consegue identificar aqueles com prognóstico reservado. Objetivo: analisar estudos envolvendo a relação entre o EEG neonatal e prognóstico neurológico, identificando características preditivas clínicas e eletroencefalográficas. Métodos: Neste artigo, foi feita uma ampla revisão da literatura envolvendo estudos a partir da década de 70 onde foi realizado EEG, no período neonatal, em neonatos de risco e/ou com crises convulsivas. Utilizou-se os bancos de dados medline, scielo e web of science. Esta revisão foi produzida no período compreendido entre janeiro e abril do ano de 2007. Resultados: O EEG pode contribuir fortemente para o estabelecimento do prognóstico e risco de óbito, principalmente quando apresenta alterações do tipo surto-supressão, isoeletricidade, baixa voltagem e dismaturidade. Em contrapartida, o exame neurológico normal e o EEG normal correlacionam-se a prognóstico mais favorável. EEGs seqüenciais fortalecem a relação com a predição do prognóstico. Conclusões: $O$ EEG é um método que possibilita a avaliação de neonatos com patologias graves podendo ser correlacionado com desfecho neurológico durante os primeiros anos de vida principalmente quando apresenta alterações do ritmo de base.
\end{abstract}

Unitermos: EEG neonatal, recém-nascidos de risco, crises convulsivas, prognóstico.

\begin{abstract}
The predictive value of neonatal EEG in high-risk newborns

Introduction: The precocious detection of cerebral dysfunction or insult in newborns of risk in the Neonatal Intensive Therapy Units increases the possibility of survival and less risk of neurological sequela in these children. The EEG is an instrument that allows to the neonatologist information about the repercussion of the neurological injury on the immature brain and, with the neuroimage findings, to identify those with reserved prognosis. Objectives: To analyse studies involving the relation between the neonatal EEG and the neurological prognosis identifying clinical and electroencephalographics predictable characteristics. Methods: we have performed a wide bibliographic revision including studies published since 1970 till now the EEG was accomplished, on the neonatal period, in neonates of risk and/or seizures. Results: EEG may help to establish outcome and mortality mainly when it presents burst suppression, inactivity and or low voltage the patterns. On the other side a normal neurological exam together with normal EEG related to a favorable outcome. Sequential EEGs also improves the sensibility to predict outcome. Conclusion: The EEG is a non-invasive low cost method that enables the evaluation of neonates with serious illness.
\end{abstract}

\footnotetext{
* Neurologista Infantil. Aluno do Pós-Graduação em Medicina e Ciências da Saúde da PUCRS.

** Acadêmicas da FAMED/PUCRS.

*** Professora Adjunta aos Departamentos de Medicina Interna/Neurologia e Pediatria da FAMED/PUCRS.

Received Oct. 25, 2007; accepted Nov. 23, 2007.
} 
The changes observed in the background activity, the presence of determined graph elements and the organization level of the sleeping stages can be correlated with the neurological outcome during the first years of life.

Key words: neonatal EEG, risk newborns, seizures, prematurity.

\section{INTRODUÇÃO}

O EEG realizado no período neonatal está firmemente estabelecido como uma técnica não-invasiva para avaliação de recém-nascidos considerados de risco. Além disso, o exame também permite o estudo de eventos ontogenéticos relacionados ao desenvolvimento normal e, sobretudo, ao desenvolvimento anormal do sistema nervoso central no período mais precoce da existência pós-natal. ${ }^{1-7}$

O EEG é indicado para a avaliação de neonatos com crises convulsivas, mostrando-se superior à avaliação clínica na detecção precoce e prognóstico da desta disfunção. ${ }^{(8-10)}$ Monod e colaboradores realizaram um dos primeiros estudos que avaliaram de forma sistemática a relação entre EEG neonatal e prognóstico de recém-nascidos. ${ }^{11}$ Muitos outros estudos enfatizaram o valor prognóstico do EEG no período neonatal. ${ }^{1-21}$ A associação do EEG envolvendo patologias graves, como as crises convulsivas ${ }^{22-29}$ e encefalopatia hipóxico-isquêmica, ${ }^{20,30-33}$ é amplamente discutida na literatura médica. Também existem estudos que determinam o valor prognóstico de anormalidades específicas dentro do período neonatal, como surto supressão, 2,34,35

Durante as últimas décadas, muitos autores abordaram esta temática. O objetivo de nosso estudo é realizar uma revisão crítica da literatura atual sobre o tema valor prognóstico do EEG neonatal em recém nascidos de alto risco.

\section{CRISES CONVULSIVAS E EEG NO PERÍODO NEONATAL}

As crises convulsivas ocorrem com maior freqüência no período neonatal do que em outras faixas etárias. Sua incidência em recém-nascidos é variável, dependendo da metodologia empregada no estudo. ${ }^{18,29}$

O EEG, no período neonatal, é o primeiro exame para identificar crises convulsivas não-detectadas clinicamente e oferece parâmetros na avaliação da lesão transitória ou permanente do sistema nervoso central. ${ }^{1-7}$ As crises convulsivas, no período neonatal, estão reconhecidamente associadas a maior risco de encefalopatia. A sua identificação nestes recém-nascidos é extremamente importante para a determinação do prognóstico, 18,29,36,37 o qual deve ser baseado na etiologia das crises, nos fatores clínicos perinatais, na evolução neurológica no período neonatal e nas características clínicas das crises convulsivas. ${ }^{18}$
O EEG tem-se tornado, nos últimos anos, uma ferramenta importante no prognóstico de recém-nascidos com crises convulsivas e, devido a sua importância na avaliação destes neonatos, está sendo utilizado cada vez mais dentro das unidades de terapia intensiva neonatal. ${ }^{1-3,7} \mathrm{~A}$ etiologia das crises, no período neonatal, pode estar relacionada a diversas patologias, tais como encefalopatia hipóxico-isquêmica, hemorragia intracraniana, infecção congênita, malformação do sistema nervoso central, distúrbios de migração neuronal, erros inatos do metabolismo, etc ${ }^{(12,18)}$. O risco de desenvolver epilepsia após a ocorrência de crises convulsivas neonatais é elevado e esta associação que pode resultar em doenças neurológicas permanentes, tais como retardo mental e paralisia cerebral, vem sendo demonstrada por diversos autores. ${ }^{27,30}$

Rose e Lombroso,em estudo envolvendo 135 recémnascidos a termo com crises convulsivas, concluíram que o EEG interictal normal após as crises convulsivas apresenta probabilidade de $86 \%$ de evolução favorável. Neste estudo, a hipoglicemia foi a principal causa das crises convulsivas neonatais. Alterações do ritmo de base como isoeletricidade, surto-supressão e baixa voltagem apresentaram prognóstico reservado. Neste estudo as crianças com EEG normal apresentaram evolução favorável. ${ }^{38}$

Vários autores analisaram a relação entre EEG e prognóstico em neonatos com crises eletrográficas e observaram que tanto o grau quanto a duração de anormalidade do ritmo de base são fatores preditivos que interferem no desenvolvimento neurológico ${ }^{(15,16)}$.

Legido e colaboradores realizaram um estudo prospectivo com 40 recém-nascidos com crises convulsivas, sendo 12 prematuros e 28 recém-nascidos a termo. A principal etiologia das crises era a asfixia perinatal seguida por meningite e disgenesia do sistema nervoso central. $\mathrm{O}$ estudo demonstrou a importância da anormalidade interictal no EEG de neonatos com crises eletrográficas para a formulação do prognóstico neurológico. Foi observada forte associação entre a anormalidade interictal (o autor não especifica o tipo de anormalidade do ritmo de base só relata anormalidade moderadamente a grave, mas não identifica qual é) do ritmo com o atraso neuropsicomotor e o desenvolvimento de epilepsia. Além disso, foi constatado um risco aumentado destes pacientes evoluírem para paralisia cerebral quando apresentavam alteração do ritmo de base moderado a grave. ${ }^{18}$ 
Da Costa e colaboradores demonstraram que as apnéias, como manifestação de crise convulsiva, apresentam prognóstico reservado, apesar das crises serem de curta duração. Todos os neonatos que apresentaram seqüelas neurológicas possuíam algum tipo de patologia neonatal associada. As principais seqüelas neurológicas encontradas foram o desenvolvimento de epilepsia e retardo no desenvolvimento neuropsicomotor. ${ }^{39}$

Nunes e colaboradores em estudo retrospectivo, constataram que neonatos com crises convulsivas que apresentam EEG sequencialmente anormal (com padrão eletrográfico de dismaturidade persistente, surto supressão e ou baixa voltagem) apresentam prognóstico neurológico desfavorável. Já os recém-nascidos com EEGs normais de forma seqüencial, geralmente, têm bom prognóstico. Nesta amostra, a etiologia mais encontrada associada à crise convulsiva foi asfixia perinatal $(52 \%) .^{3}$

Em outro estudo realizado pelo mesmo grupo com neonatos com estado de mal convulsivo, a atividade ictal mais prevalente foi a atividade rítmica pseudofisiológica e a interictal foi a atividade multifocal, ${ }^{35}$ sendo o prognóstico associado a alterações do ritmo de base e ao diagnóstico etiológico.

McBride e colaboradores analisando a duração, o número e a quantificação das crises eletrográficas em recémnascidos, comparou o prognóstico dos mesmos com e sem crises eletrográficas, observando a existência de uma relação entre a freqüência de crise eletrográfica com morbidade e mortalidade, além de uma forte relação com a etiologia asfixia perinatal. ${ }^{17}$

Oliveira e colaboradores estudaram as descargas de breve duração e concluiram que estas contribuem para o diagnóstico de crises convulsivas no período neonatal, sendo correlacionadas com risco aumentado para prognóstico neurológico reservado. A atividade rítmica pseudofisiológica foi observada em $54,5 \%$ dos recém-nascidos com suspeita de crise convulsiva. ${ }^{6}$

Mizrahi e colaboradores (1987) detectaram que crises eletrográficas sem manifestação clínica apresentavam prognóstico reservado nos recém-nascidos que foram acompanhados durante dois anos. ${ }^{28}$

Da Silva e colaboradores estudaram a relação entre neonatos que apresentaram convulsões e o risco de desenvolvimento de epilepsia. A incidência de epilepsia foi de $22 \%$ aos 12 meses e 28,6\% aos 36 meses, onde o EEG interictal normal e o exame neurológico normal foram relacionados de forma significativa com prognóstico favorável e com a ausência de epilepsia. ${ }^{27}$

Tekgul e colaboradores demonstraram que padrões específicos do EEG, em neonatos com crises convulsivas, têm valor preditivo precoce e são mais confiáveis para o prognóstico neurológico tardio. $\mathrm{O}$ fator preditivo mais im- portante relacionado com o desenvolvimento neuropsicomotor relaciona-se com a etiologia das crises neonatais e com a anormalidade moderado a grave do ritmo de base. $^{12}$

Analisando o valor prognóstico do EEG com surto supressão não reativo associado a convulsões neonatais, os autores concluíram que este padrão está associado a inúmeras desordens neurológicas durante o período neonatal e também a crises convulsivas precoces e refratárias, além de morbidade e mortalidade elevadas. ${ }^{2,35}$

Kumar e colaboradores analisaram características clínicas, etiológicas e eletroencefalográficas em recém-nascidos com crises convulsivas. A etiologia principal foi asfixia perinatal, onde o EEG destas crianças com este diagnóstico apresentava-se anormal em 26,7\%. As crianças com hemorragia intracraniana apresentaram-se na maioria com padrão de surto-supressão (66\%). Anormalidades eletroencefalográficas foram encontradas em 33,4\% das crianças estudadas. ${ }^{40}$

Vários estudos enfatizam a importância da monitorização contínua do EEG dentro das unidades de terapia intensiva neonatal em recém-nascidos graves com crises convulsivas de difícil controle, asfixia perinatal e prematuridade. A monitorização prolongada de 24-72 horas influencia no prognóstico à longo prazo, pois ajuda na identificação precoce de anormalidade de ritmo de base, anormalidades na estruturação do sono e de crises sutis não identificadas clinicamente. . $^{7,30,41,42}$

\section{EEG NEONATAL EM RECÉM-NASCIDOS DE RISCO}

Monod e colaboradores, avaliando 240 recém-nascidos a termo e prematuros, observaram que o EEG seqüencial tem mais sensibilidade para definir prognóstico. Constataram que os padrões de inatividade do ritmo de base e surto supressão, mesmo em um único exame, correlacionam-se com prognóstico reservado. Definiram a primeira semana de vida como o melhor momento para realizar o EEG com o objetivo de estabelecer o prognóstico. ${ }^{11}$

Tharp e colaboradores, estudando neonatos prematuros com padrões de inatividade do ritmo de base, sutosupressão, descontinuidade excessiva e ondas agudas positivas, constataram que essas crianças evoluíram para mau prognóstico. ${ }^{9}$

Holmes e Lombroso observaram que alterações do ritmo de base são mais importantes para a correlação com prognóstico do que atividade epileptiforme. Concluíram que exames seqüenciais aumentam o poder preditivo do EEG neonatal, onde padrões anormais do tipo inatividade do ritmo de base, surto-supressão mesmo em exame isolado relacionam-se a prognóstico reservado. $^{21}$ 
Nunes e colaboradores, avaliando 85 prematuros que apresentaram patologias graves no período neonatal, observaram que a alteração eletroencefalográfica mais encontrada nos recém-nascidos com asfixia e desnutrição intrauteriana foi padrão dismaturo da atividade elétrica cerebral. Naqueles neonatos com crises convulsivas, a alteração mais freqüente foi anormalidade paroxística anormal (ondas agudas positivas) e imaturidade. Os achados eletroencefalográficos associados com mau prognóstico foram isoeletricidade e atividade paroxística anor$\mathrm{mal}^{4}$

Em estudo prospectivo avaliando a evolução das ondas agudas frontais, grafoelemento fisiológico do EEG neonatal, foi observado que as ondas agudas frontais podem ser relacionadas com a maturidade bioelétrica, pois são mais prevalentes em recém-nascidos a termo ou próximos ao termo e devem desaparecer em torno de 44-45 semanas de idade concepcional. ${ }^{35}$ Os mesmos autores também observaram uma tendência das ondas agudas frontais ocorrerem de forma síncrona e bilateral em neonatos a termo, diferente do encontrado em prematuros. $^{43}$

Avaliando a bioletrogenese das ondas agudas temporais em EEG de recém-nascidos a termo e prematuros, os autores observaram que as ondas agudas temporais ocorrem em neonatos normais e sua incidência varia de acordo com a morfologia e tende a desaparecer com o aumento da idade concepcional. ${ }^{44}$

A obtenção de EEG/polissonografia em recém-nascidos com asfixia perinatal permite a detecção de elementos subclínicos, como o padrão de inatividade do ritmo de base, sendo este foi o padrão anormal mais freqüentemente encontrado nos neonatos com asfixia. Estes achados possibilitam a identificação de disfunção cerebral, sendo possível relacioná-los com critérios prognósticos a longo prazo. A precocidade da obtenção do registro eletroencefalográfico possibilitará a deteç̧ão de anormalidades mínimas que podem passar despercebidas. ${ }^{45}$

A asfixia perinatal é a causa mais freqüentemente associada a convulsões no período neonatal. ${ }^{3,12,17,18,27,30,40} \mathrm{Em}$ vários estudos, a sua incidência é de 15 a $53 \% .^{12,18,30,40} \mathrm{Em}$ estudo recente, o EEG de 33,34\% dos recém-nascidos com asfixia perinatal apresentava-se anormal, onde as anormalidades mais frequentemente encontradas foram surto-supressão, atividade multifocal, dismaturidade, atenuação do ritmo de base e isoeletricidade. ${ }^{40}$

A relação entre asfixia perinatal e padrão de surto supressão foi recentemente estudada. Os autores observaram que, em neonatos que realizaram EEG precocemente em até 03 horas de vida, foi identificado o surgimento de surto-supressão. Quando o EEG era realizado em até 6 horas de vida, além de surto-supressão, também foi encontrada baixa voltagem, sendo essas anormalidades asso- ciadas a um prognóstico bastante reservado e existindo forte relação entre surto supressão e asfixia perinatal durante o período neonatal. ${ }^{33}$

Recente estudo retrospectivo, in press, avaliou a relação do EEG seqüencial e o prognóstico neurológico em recém-nascidos com crises convulsivas, a fim de identificar fatores preditivos neurológicos. $\mathrm{O}$ estudo envolveu 58 recém-nascidos com crises convulsivas de 02 coortes históricos. A anormalidade do ritmo de base seqüencial foi relacionada com atraso de desenvolvimento neuropsicomotor e epilepsia. Surto supressão quando presente estava associada com epilepsia e óbito pós-neonatal. A anormalidade do ritmo de base seqüencial no primeiro e segundo EEGs apresentou um risco aumentado para epilepsia e atraso de desenvolvimento neuropsicomotor. A anormalidade do ritmo de base em pelo menos um dos EEGs, quando realizados sequencialmente, foi mais importante para determinar prognóstico neurológico do que anormalidade ictal ou anormalidade na organização do sono. ${ }^{46}$

Scher, em uma ampla revisão sobre EEG neonatal estabelece de forma didática o padrão evolutivo deste exame de 28 até $38-42$ semanas de idade gestacional. O autor enfatiza a importância da realização de EEGs seqüenciais para melhor acurácia no estabelecimento do prognóstico neurológico. ${ }^{47}$

\section{CONCLUSÃO}

Concluindo, é possível afirmar que o EEG neonatal trouxe grande contribuição para o estudo de recém nascidos de alto risco, facilitando o reconhecimento de manifestações clínicas sutis, eventualmente não identificadas clinicamente. A associação do exame de imagem com o EEG pode contribuir fortemente para o estabelecimento do prognóstico e risco de óbito, principalmente quando apresenta alterações do tipo surto-supressão, isoeletricidade, baixa voltagem e dismaturidade. Em contrapartida, o exame neurológico normal e o EEG normal correlacionam-se a prognóstico mais favorável. EEGs seqüenciais e a identificação de padrão específico em pelo menos um dos EEGs, como surto-supressão, são importantes para predizer o prognóstico. A monitorização contínua ou prolongada do neonato de risco dentro da unidade de terapia intensiva neonatal é de suma importância para avaliação de anormalidades específicas, subclínicas, que podem corroborar com piora do prognóstico e que sendo diagnosticadas podem ser tratadas. A asfixia perinatal persiste sendo a principal causa associada a crises convulsivas precoces, nos estudos revisados. Os EEGs destas crianças quando apresentam-se com alterações específicas do ritmo de base, como surto-supressão e anormalidade multifocal, relacionam-se a prognóstico neurológico desfavorável a longo prazo. 
Valor prognóstico do EEG neonatal em recém-nascidos ...

\section{REFERÊNCIAS}

1. Laroia N, Guillet R, Burchfiel J, McBride MC. EEG background as predictor of electrographic seizures in high-risk neonates. Epilepsia. 1998;39:545-51.

2. Menache CC, Bourgeois BF, Volpe JJ. Prognostic value of neonatal discontinuous EEG. Pediatr Neurol. 2002;27:93-101.

3. Nunes ML, Costa JC, Godinho CC, Rodrigues MP. Prognóstico de recém-nascido com convulsões: aspectos clínicos e eletroencefalográficos. J Liga Bras Epilepsia. 1994;7:27-30.

4. Nunes ML, Costa JC, Taufer L, da Silveira CM. Valor do EEG na caracterização e prognóstico de patologias neurológicas em recémnascidos prematuros. Arq Neuropsiquiatr. 1995;53:625-30.

5. Nunes ML, Costa JC. Manual de EEG e polissonografia neonatal: atlas de traçados. Porto Alegre: EDIPUCRS; 2003.

6. Oliveira AJ, Nunes ML, Haertel LM, Reis FM, da Costa JC. Duration of rhythmic EEG patterns in neonates: new evidence for clinical and prognostic significance of brief rhythmic discharges. Clin Neurophysiol. 2000;111:1646-53.

7. Tharp BR. Intensive video/EEG monitoring of neonates. Adv Neurol. 1987;46:107-26.

8. Lombroso CT. Neonatal EEG polygraphy in normal and abnormal newborns. In: Niedermeyer E, Silva FL, editors. Electroencephalography: basic principles, clinical applications, and related fields. $3^{\text {rd }}$ ed. Baltimore: Williams \& Wilkins; 1993. p.803-75

9. Tharp BR, Scher MS, Clancy RR. Serial EEGs in normal and abnormal infants with birth weights less than 1200 grams - a prospective study with long term follow-up. Neuropediatrics. 1989;20:64-72.

10. Scher MS, Aso K, Beggarly ME, Hamid MY, Steppe DA, Painter MJ. Electrographic seizures in preterm and full-term neonates: clinical correlates, associated brain lesions, and risk for neurologic sequelae. Pediatrics. 1993;91:128-34.

11. Monod N, Pajot N, Guidasci S. The neonatal EEG: statistical studies and prognostic value in full-term and pre-term babies. Electroencephalogr Clin Neurophysiol. 1972;32:529-44.

12. Tekgul H, Gauvreau K, Soul J, Murphy L, Robertson R, Stewart J, Volpe J, Bourgeois B, du Plessis AJ. The current etiologic profile and neurodevelopmental outcome of seizures in term newborn infants. Pediatrics. 2006;117:1270-80.

13. Rowe JC, Holmes GL, Hafford J, Baboval D, Robinson S, Philipps A, Rosenkrantz T, Raye J. Prognostic value of the electroencephalogram in term and preterm infants following neonatal seizures. Electroencephalogr Clin Neurophysiol. 1985;60:183-96.

14. Bye A, Flanagan D. Electroencephalograms, clinical observations and the monitoring of neonatal seizures. J Paediatr Child Health. 1995;31:503-7.

15. Eyre JA, Wilkinson AR. Adverse perinatal factors predictive of clinical and subclinical seizure in the newborn [abstract]. Neuropediatrics. 1987;18:125.

16. Connell J, Oozeer R, de Vries L, Dubowitz LM, Dubowitz V. Continuous EEG monitoring of neonatal seizures: diagnostic and prognostic considerations. Arch Dis Child. 1989;64:452-8.

17. McBride MC, Laroia N, Guillet R. Electrographic seizures in neonates correlate with poor neurodevelopmental outcome. Neurology. 2000;55:506-13.

18. Legido A, Clancy RR, Berman PH. Neurologic outcome after electroencephalographically proven neonatal seizures. Pediatrics. 1991;88:583-96.

19. Watanabe K, Kuroyanagi M, Hara K, Miyazaki S. Neonatal seizures and subsequent epilepsy. Brain Dev. 1982;4:341-6.

20. Holmes G, Rowe J, Hafford J, Schmidt R, Testa M, Zimmerman A. Prognostic value of the electroencephalogram in neonatal asphyxia. Electroencephalogr Clin Neurophysiol. 1982;53:60-72.
21. Holmes GL, Lombroso CT. Prognostic value of background patterns in the neonatal EEG. J Clin Neurophysiol. 1993;10:323-52.

22. Lombroso CT. Prognosis in neonatal seizures. Adv Neurol. 1983;34:101-13.

23. Lombroso CT. Neonatal seizures: a clinician's overview. Brain Dev. 1996;18:1-28.

24. Holden KR, Mellits ED, Freeman JM. Neonatal seizures. I. Correlation of prenatal and perinatal events with outcomes. Pediatrics. 1982;70:165-76.

25. Painter MJ, Bergman I, Crumrine P. Neonatal seizures. Pediatr Clin North Am. 1986;33:91-109.

26. Andre M, Matisse N, Vert P, Debruille C. Neonatal seizures: recent aspects. Neuropediatrics. 1988;19:201-7.

27. Garcias da Silva LF, Nunes ML, Costa JC. Risk factors for developing epilepsy after neonatal seizures. Pediatr Neurol. 2004;30:271-7.

28. Mizrahi EM, Kellaway P. Characterization and classification of neonatal seizures. Neurology. 1987;37:1837-44.

29. Volpe JJ. Neonatal seizures. In: Neurology of the newborn. $4^{\text {th }}$ ed. Philadelphia: Saunders; 2001.p.178-214.

30. Murray DM, Ryan CA, Boylan GB, Fitzgerald AP, Connolly S. Prediction of seizures in asphyxiated neonates: correlation with continuous video-electroencephalographic monitoring. Pediatrics. 2006;118:41-6.

31. Pressler RM, Boylan GB, Morton M, Binnie CD, Rennie JM. Early serial EEG in hypoxic ischaemic encephalopathy. Clin Neurophysiol. 2001;112:31-7.

32. Coen RW, McCutchen CB, Wermer D, Snyder J, Gluck FE. Continuous monitoring of the electroencephalogram following perinatal asphyxia. J Pediatr. 1982;100:628-30.

33. Shany E, Goldstein E, Khvatskin S, Friger MD, Heiman N, Goldstein $\mathrm{M}$, et al. Predictive value of amplitude-integrated electroencephalography pattern and voltage in asphyxiated term infants. Pediatr Neurol. 2006;35:335-42.

34. Al-Futaisi A, Banwell B, Ochi A, Hew J, Chu B, Oishi M, et al. Hidden focal EEG seizures during prolonged suppressions and highamplitude bursts in early infantile epileptic encephalopathy. Clin Neurophysiol. 2005;116:1113-7.

35. Nunes ML, Giraldes MM, Pinho AP, Costa JC. Prognostic value of non-reactive burst suppression EEG pattern associated to early neonatal seizures. Arq Neuropsiquiatr. 2005;63:14-9.

36. Costa JC, Nunes ML, Fiori RM. Seizures in the neonatal period. J Pediatr (Rio J). 2001; 77 Suppl 1:S115-22.

37. Nunes ML, Costa JC Outcome of newborns with neonatal seizures: risk factors and outcome. Curr ped rev 2006; 2: 315-21.

38. Rose AL, Lombroso CT. A study of clinical, pathological, and electroencephalographic features in 137 full-term babies with a long-term follow-up. Pediatrics. 1970;45:404-25.

39. Da Costa JC, Nunes ML, Nora DB, Lopes DK, Becker J. Apnéia como manifestação convulsiva sutil em neonatos: investigação clínica e valor prognósticoe diagnóstico da polissonografia. Jornal da Liga Brasileira de Epilepsia. 1991;4:17-21.

40. Kumar A, Gupta A, Talukdar B. Clinico-etiological and EEG profile of neonatal seizures. Indian J Pediatr. 2007;74:33-7.

41. Hellström-Westas L. Continuous electroencephalography monitoring of the preterm infant. Clin Perinatol. 2006;33: 633-47.

42. Rosén I. The physiological basis for continuous electroencephalogram monitoring in the neonate. Clin Perinatol. 2006; 33:593-611.

43. Nunes ML., Da Costa JC, Becker J. Distribution of frontal sharp transients in healthy and high risk newborns. Brazilian Journal of Epilepsy and Clinical Neurophysiology. 1998;4:7-11. 
44. Nunes ML, Gameleira FT, Oliveira AJ, Da Costa JC. Developmental characteristics of temporal sharp transients in the EEG of normal preterm and term newborns. Arq Neuropsiquiatr. 2003;61:574-9.

45. Pozo PL, Camejo AP. Importance of the electroencephalogram in perinatal asphyxia. Rev Cubana Pediatr. 1995;67:71-6.

46. Khan RL, Nunes ML, Garcias LF, Da Costa JC. Predictive value of sequencial electroencephalography (EEG) in neonates with seizures and its relation to neurological outcome. Journal of Child Neurology. In press.
47. Scher MS. Eletroencephalography of the newborn: Normal and abnormal features. In: Eletroencephalography. Basic principles, clinical applications and related fields. $5^{\text {th }}$ ed. Lippincott Williams \& Wilkins; 2005. p.896-946.

\section{Endereco para correspondência:}

Magda Lahorgue Nunes

Serviço de Neurologia do HSL-PUCRS

Av. Ipiranga 6690 sala 220

Fone/Fax: (51) 3339-4936

E-mail: nunes@pucrs.br 